\title{
Intention d'usage des messages réseau-publicitaires par le cybernaute nord-africain : Etude qualitative
}

\author{
Hathout Chaimae ${ }^{1}$, Hamadi Chakib ${ }^{2}$, and Hathout Sara ${ }^{3}$ \\ ${ }^{l}$ Docteur en sciences de gestion à LAREGO ENCG université Cadi Ayyad, Marrakech, Maroc \\ ${ }^{2}$ Professeur de l'Enseignement Supérieur -Phd ENCG université Cadi Ayyad, Marrakech, Maroc \\ ${ }^{3}$ Doctorante en sciences de gestion à LSEPP FSEJS université Ibn Tofail, Kénitra, Maroc
}

\begin{abstract}
Résumé : Ces dernières années, imprégnées d'une large démocratisation de la publicité sur les réseaux sociaux, nous cherchons à modéliser la relation entre les stratégies de communication électronique menées sur ces plateformes par une entreprise pratiquant le Yield management, et l'attitude du cybernaute nord-africain envers cette forme de publicité sociale. Dans ce cadre, un modèle de recherche a été élaboré au fur et à mesure que nous avancions dans notre littérature. Nous avons ensuite cherché à analyser et contextualiser les différents éléments dudit modèle à travers une étude qualitative.
\end{abstract}

Mots-clés : communication électronique, réseaux sociaux, publicité électronique, réseau-publicité, yield Management, les nouvelles aides à la vente et à l'achat (NAVAs).

\section{INTRODUCTION}

«It takes 20 years to build a reputation and 5 minutes to ruin it », une maxime avancée par le bien connu américain Warren Buffet, et qui se manifeste dans toute son ampleur à l'ère des réseaux sociaux, où une information - même infondée, peut se propager et induire de sérieux dommages, ou à contrario conduire au triomphe. Ces dernières années au Maroc, nous avons tous été témoins de controverses de tous genre initiées sur les R.S. (réseaux sociaux). Cet activisme cybernétique a fini par rattraper le champ économique en pesant encore et toujours sur le rendement de firmes nationales tel qu'Afriquia et les eaux minérales d'Oulmes après le mouvement du boycott et avait pénalisé la centrale laitière Danone bien avant avec l'amalgame de « Raibi Jamila » en 2015. Sur un registre plus optimiste, ces mêmes plateformes ont pu conduire à des triomphes médiatiques et économiques telles que «Sananas Beauty » et la campagne du «Count Chiquinho Scarpa ». A tout ceci s'ajoute la pandémie actuelle du coronavirus qui par la distanciation sociale, le confinement et toutes ses restrictions sanitaires n'a fait que confirmer le grand rôle que joue ses plateformes sociales comme un canal efficace de communication.

Cette communication sur les R.S., surtout commerciale, et son efficacité ne sont plus sujettes au tâtonnement et devraient faire l'objet de réflexions poussées aussi bien au niveau professionnel qu'académique. Ainsi le webmarketing s'est vu être une suite logique de l'émergence d'Internet, une mutation révolutionnaire du marketing traditionnel qui s'est rapidement vêtue du cap du commun illustrant l'essor perpétuel de ce bassin technique et technologique irriguant sans cesse le domaine publicitaire en général et apprêtant à l'avènement de la «Social-publicité » en particulier. Le côté hédonique et ludique de ce nouveau 
moyen de communication a sans doute bouleversé la donne de la publicité classique et de son efficacité, poussant la communauté scientifique à s'interroger si nous devrions toujours approcher l'efficacité publicitaire de la même manière et selon le même paradigme.

Un autre constat est que dans un monde ou les frontières internationales ne sont plus que traits dessinés sur des cartes, des géants œuvrant dans le secteur touristique ou le transport aérien, entre autres, tirent bien profit de cette dématérialisation offerte par l'écosystème digital. Il s'agit des entreprises pratiquant le Yield management et dont le système tarifaire dynamique fait qu'elles éprouvent une grande nécessité de vélocité et d'instantanéité de part leur communication; un besoin assouvi en grande partie par l'usage des médias sociaux.

De tout ce qu'on vient d'avancer on pourrait dire qu'il serait obsolète de parler d'efficacité publicitaire sans se référer au rôle que joue le Web et particulièrement les réseaux sociaux dans ce domaine. Ainsi nous proposons d'examiner «l'impact des stratégies de communication électronique conduites via réseaux sociaux, sur « l'efficacité réseau-publicitaire » des entreprises pratiquant le Yield mangement. »

Pour creuser la chose, nous décortiquons notre problématique en trois sous-questions phares :

- Quelles sont les principales stratégies de communication électronique utilisées sur les réseaux sociaux?

- Quel en est l'impact sur l'efficacité réseau-publicitaire d'une entreprise ?

- Quelles sont les dimensions susceptibles de régir et / ou agir sur le lien entre ces deux variables ?

Pour donner réponse à nos questions de recherche, une consultation de la littérature a été faite pour dresser notre cadre théo-conecptuel, suivie par une étude qualitative de contextualisation. Pour ce faire, et comme le veut la coutume, nous commencerons d'abord par s'arrêter sur nos cadres conceptuel et théorique, on s'attardera par la suite sur les hypothèses et modèle primaires, la méthodologie de travail utilisée sur toutes les étapes de notre étude empirique, nous exposerons et discuterons les résultats obtenus pour finir sur les apports majeurs, les limites et les perspectives de la recherche.

\section{CADRE CONCEPTUEL DE LA RECHERCHE :}

Nous commençons ici par définir nos concepts phares et soulignons que le choix des définitions adoptées s'est fait sur deux principes : généralité et contexte :

- Communication électronique (Zlitini, 2012) «Mutation des téléinformatiques causée par la convergence des technologies, des télécommunications, de l'informatique et des multimédias ».

- Publicité électronique (IAB Europe, 2010) «La publicité en ligne s'entend de l'utilisation d'Internet comme moyen publicitaire pour publier ses annonces sur différents sites et dans les pages de résultats des moteurs de recherche ».

- Réseaux sociaux (Thiers, 2013) « Des plateformes dont la vocation première est la mise en relation des utilisateurs entre eux ». Distinction doit être faite entre les R.S. (EX : Facebook ; Instagram ; Youtube...) fondés essentiellement sur le partage et l'interaction et les médias sociaux (Blogs, newsletters...) qui ont une portée plus générale et orientée inbound et diffusion de contenu.

- Efficacité publicitaire «L'efficacité publicitaire mesure la capacité d'une campagne ou d'un message à atteindre les objectifs de l'annonceur. » 
- Yield management (Capiez, 2003; Lovelock et Wirtz, 2005) «Une approche de communication personnalisée, programmée à maximiser le profit de l'entreprise tout en réformant le service client. »

- Réseau-Publicité : (nous même,2020) Se réfère à l'ensemble des opérations d'émission et de diffusion publicitaire de contenus sur les différents réseaux sociaux. Distinction doit être faite entre « réseau-publicité » et un « réseau publicitaire Internet» qui est constitué d'un ensemble de sites ou d'applications mobiles regroupés dans le cadre d'une offre publicitaire plus ou moins homogène ; et entre réseau-publicité et «publicité sociale » qui désigne l'usage des différents médias sociaux pour effectuer des communications marketing au profit de la clientèle cible. Par extension nous considérons que l'efficacité réseau-publicitaire mesure l'efficacité du contenu partagé par l'annonceur sur ses comptes et pages réseaux sociaux et non pas sur tous les médias sociaux.

En explorant la littérature, nous avons fait quelques constats majeurs. Le premier est que par l'usage des NAVs (les Nouvelles Aides à la Vente est un ensemble de moyens mis à la disposition du client pour l'assister dans son processus de prise de décision. Ex : agent virtuel intelligent; réalité augmentée; intelligence artificielle ; Adver-gaming; comparateur de prix ;...) la publicité digitale s'annonce comme étant un moyen ludique réconciliant les prescriptions managériales et les exigences des consommateurs devenus friands de nouveautés et de plus en plus avertis. Cette forme de publicité recèle un nombre d'avantage allant du gain de temps à la dématérialisation des procédés en passant par cette emprise qu'elle a sur de la distance et le rôle de mine informationnelle qu'elle assure.

Le deuxième constat structurel est que les annonceurs ont en effet finis par comprendre que leurs messages e-publicitaires se devaient d'être plus personnels au moment même où l'e-publicité à travers les réseaux sociaux permet d'un côté aux internautes d'assouvir leur besoin d'appartenance et garantit un lien plus intime allant même jusqu'à la coproduction de contenu. L'avènement de cette forme de publicité électronique, a donné lieu à l'apparition d'un nouveau processus de décision collective qu'on qualifie de «cross canal communautaire ». Il s'agit d'une situation dans laquelle les membres d'une même communauté activent plusieurs canaux, collectivement, dans le cadre d'un même processus de décision. Cette interaction entre consommateurs-internautes durant le processus d'achat doit amener les marques à penser « one to ones » au lieu de « one to one » et donc de penser CoRM (community relationship management) : gestion de la relation communautaire, au lieu de CRM (Customer relationship management) : gestion de la relation client. L'idée n'est donc pas seulement d'être présent sur ces plateformes, mais de maîtriser l'efficacité de sa présence. Sago (2011) et Fogel (2014) avancent que les réseaux sociaux permettent d'accroître l'efficacité de la circulation d'un message publicitaire. Maubisson (2015), lui, avance que l'efficacité de la présence d'une marque sur les réseaux sociaux dépend de sa gestion des quatre composantes du CoRM ou ce qu'on appel les 4C en «social-marketing» (communauté, connexion, conversation, contenu). Selon Abaidi (2018), Il est possible de considérer que l'efficacité publicitaire passe par le contenu informationnel du message. En plus de Ranchoux (2016), ces deux derniers auteurs, s'accordent sur le fait que l'efficacité d'existence d'une entreprise dépend essentiellement de la qualité des actualités et messages publiées et la qualité d'interaction avec les internautes. Contrairement à ces auteurs P Bagozzi (2007) analyse l'efficacité publicitaire en aval. Il stipule que l'efficacité publicitaire d'un message à travers un réseau social passe par trois éléments clefs : La qualité de réponse aux interventions des internautes; le traitement des demandes des internautes; et le contrôle du Bashing : Le Bashing social désigne la diffamation communautaire à l'égard d'une marque (d'un produit, d'une personne, d'un sujet...) sur ces plateformes sociales. 
Le troisième constat se voit être la réponse à notre lére question de recherche. En effet, Entre raisonnements et capacités, technologies et pratiques, deux dimensions réunissent les stratégies de communication électronique : la dimension «Marque » réunissant la stratégie de positionnement et la stratégie de qualité, et la dimension «Consommateur» avec la stratégie d'interaction et la stratégie de réplique. De chaque stratégie, ont été prélevés les éléments propres à la communication sociale.

Ainsi, dans le cadre de la stratégie de «réseau-positionnement», deux concepts majeurs sont répétitivement cités et étudiés par différents auteurs : il s'agit de la légitimité d'existence et du caractère intrusif perçu. A cet effet, Beck et Crié (2018) démontrent que dans le cas particulier des réseaux sociaux, le caractère intrusif perçu d'un message publicitaire est directement et négativement corrélé à la légitimité d'existence de la marque ou de l'entreprise sur le réseau social. Ainsi plus l'annonceur dispose d'une bonne stratégie de « réseau-positionnement» légitimant sa présence sur un réseau social, moins le caractère intrusif perçu pourrait être fort et plus l'individu pourrait être amené à interagir avec la publicité.

En ce qui est de la stratégie de qualité, Bousquet (2015), courbet (2005) et Barber (2010) précisent qu'en communication sociale, la stratégie de qualité s'articule autour de la qualité des publications des marques qui elle-même est conditionnée par l'usage des NAVs qui concourent à des fonctions hédoniques comme : séduire le consommateur, apporter un « fun factor », optimiser l'inspiration du client, et susciter l'envie d'acheter.

Pour ce qui est de la stratégie d'interaction appartenant à la dimension consommateur, SALLARD (2007), Boucher (2009), Rocheleau (2020), Valin (2012), tous stipulent que l'interaction sur les réseaux sociaux se matérialise par l'intérêt ou l'attention exprimée à l'égard des internautes à travers le discours et/ou les procédés des cyber-annonceurs.

Le quatrième élément qui clôture la liste des antécédents à l'efficacité réseau-publicitaire dans le cadre de notre recherche, est relevé de la stratégie de réplique. Ici, Richard P Bagozzi (2007) s'attarde sur la stratégie de réplique dans un contexte de medias sociaux et stipulent qu'une bonne stratégie de réplique sur un réseau social passe essentiellement par la qualité de réponse et le traitement des demandes des internautes.

Après avoir établi la liste primaire des antécédents à l'efficacité réseau-publicitaire et donc avoir défini nos élément exogènes et par le même biais un élément modérateur qu'est l'usage des NAVS, on se réfère au modèle d'acceptation des nouvelles technologies: le TAM, lui-même fondée sur la théorie de l'action raisonnée, pour compléter les autres niveaux de notre propre modèle. La raison du recours au TAM pour l'efficacité en réseau-publicité provient de l'explication fournie par Bauer (2005) qui considère que la publicité digitale représente un cas particulier d'application du TAM à savoir une innovation d'usage : « la communication d'un contenu via un média digital ne peut être efficace qu'à condition que les consommateurs tolèrent la réception régulière de messages publicitaires sur ce media ». Nous suivons aussi les pas de Herault (2010) et de Ben Brahim (2015) qui ont adopté la même approche mais cette fois-ci dans le contexte de la publicité mobile (sur téléphone portable). Il est intéressant de citer que cette forme de publicité (publicité mobile) appartenant au marketing mobile, avec la forme que nous étudions (réseau-publicité) et qui appartient au marketing de réseau, constituent tous deux des piliers du webmarketing intelligent dit aussi 3.0. 


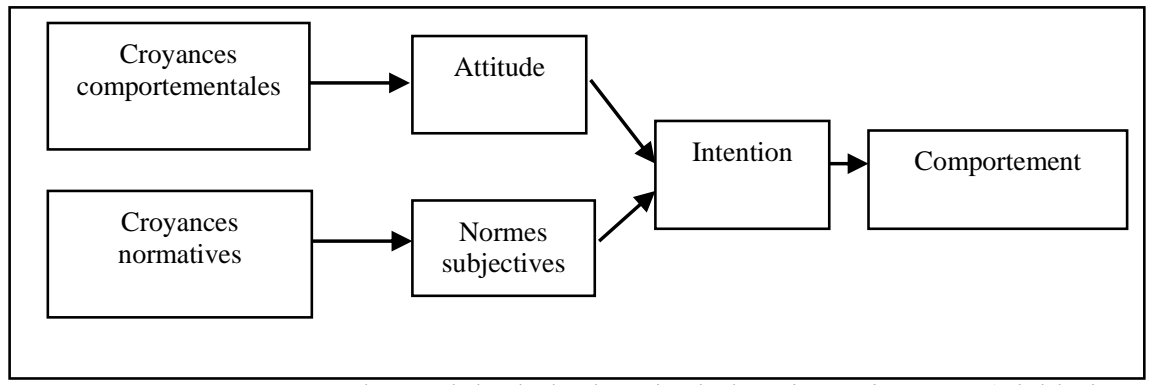

Figure 1. Représentation du modèle de la théorie de l'action raisonnée (Fishbein et Adjzen, 1975)

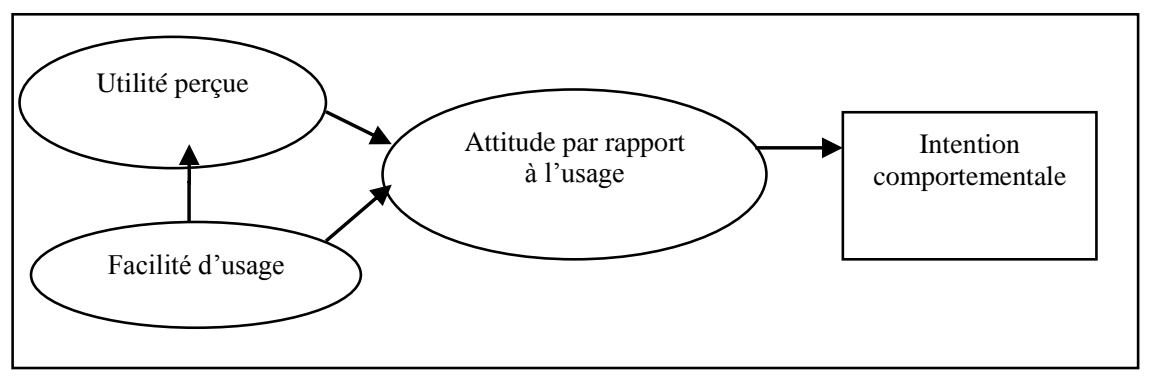

Figure 2. Représentation du modèle TAM (Technology Acceptance Model) (Venkatesh et al., 2003)

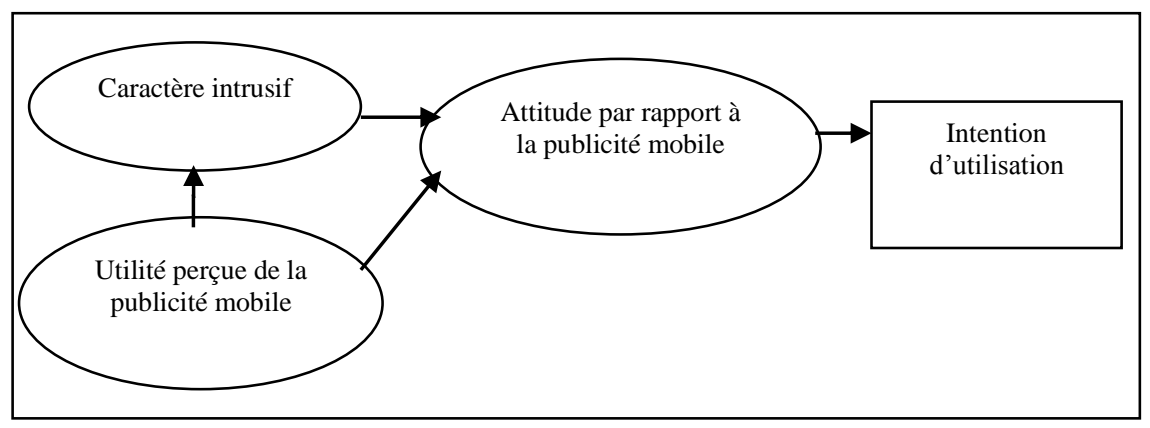

Figure 3. Représentation du modèle Herault de l'efficacité publicitaire mobile (2010).

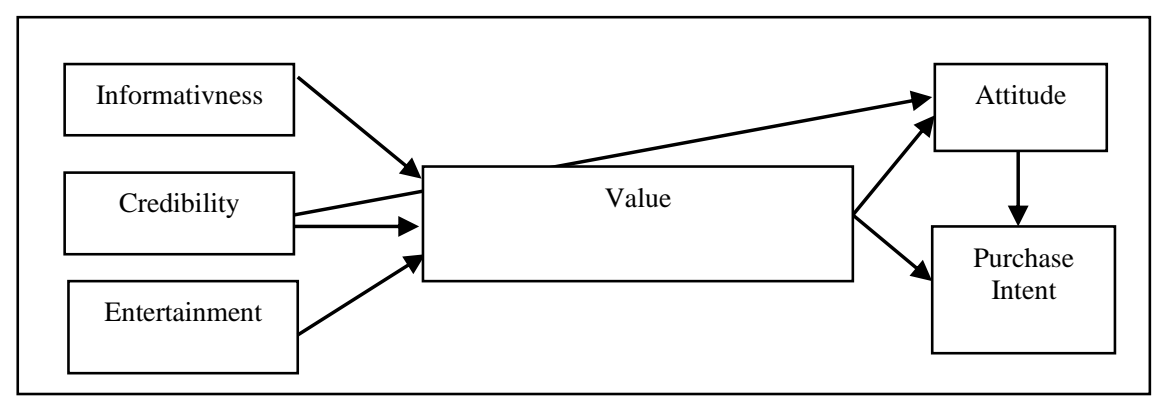

Figure 4. Représentation du modèle de Ben Brahim (2015). 
Par ce dernier constat théorique, nous aurions défini notre cadre conceptuel et nous nous arrêtons par la suite sur la contextualisation théorique de notre recherche.

A. Cadre théorique de la recherche :

Le premier constat fait à ce niveau serait que le recours au Yield Management comme terrain d'étude revient au shifting qu'à connu la nature même de ce système de part sa définition et ses composantes. Dès lors, le Yield management ne se restreint plus à un système informatique ou un nombre de techniques mathématiques, il se voit devenir une approche de communication personnalisée avec des principes de rentabilité et de maximisation de revenu. Cette nouvelle vision relationnelle du Yield management, ne s'arrête pas à sa définition, bien au contraire, elle s'enracine dans les éléments fondamentaux de ce système à savoir la tarification, la segmentation et la prévision. Ainsi et comme le ressort Zrelli (2008), la dimension relationnelle recentre le lien entre la tarification dynamique et la communication personnalisée autour de trois éléments : la synchronisation, l'individualisation et la durabilité.

Le deuxième constat ici est que quoi que la nature du lien entre Yield management et usage des réseaux sociaux reste controversé, l'existence même de ce lien est admises par plusieurs auteurs. En effet, des auteurs tel que Sigala, Christou et Gretzel (2012); Anderson (2016); Blal et Sturman (2017) et bien d'autres s'arrêtent sur les effets positifs qu'exercent l'usage des réseaux sociaux sur les pratiques de la tarification dynamique alors que des auteurs tels que Cooper, Mello et Kleywegt (2016) ; Noone, McGuire et Rohlfs (2011) se retournent vers la portée contraignante qu'ont ces médias sur les entreprises opérant par Yield Management.

Avec cette déclaration, on aurait dressé notre cadre théo-conceptuel et délimiter notre contexte théorique. Nous passons directement à l'exposition de nos hypothèses de recherche et notre modèle conceptuel de départ.

B. Hypothèses de recherche et modèle conceptuel primaire :

Et donc après avoir balayé la revue de littérature, et sur la base du modèle TAM et dans le cadre de la modélisation de l'impact de la communication sociale sur l'efficacité réseau-publicitaire, nous cherchons à identifier et mesurer l'importance respective des déterminants présupposés à l'acceptation de cette innovation d'usage.

L'objectif de notre travail de recherche est de tester un modèle dérivé du TAM en intégrant des construits pertinents lorsqu'il s'agit de réseau publicité : la légitimité d'existence sur un réseau social, la qualité des publications et messages émis sur les réseaux sociaux, l'attention portée aux internautes par les annonceurs, et la qualité des réponses aux interventions des internautes. Ces quatre éléments constituent les caractéristiques exogènes de notre modèle et incarnent le stade cognitif de la hiérarchie des effets. Nous évaluerons par la suite l'influence de chacun de ces antécédents sur l'attitude envers la réseau-publicité qui incarne ici le stade affectif de ce modèle. Cet élément représente un médiateur pré-conatif à l'efficacité réseau-publicitaire.

Sachant que comme le caractère intrusif, la légitimité d'existence perçue est un élément d'ordre cognitif de la réseau-publicité, et suivant les travaux d'Abaidi et al. (2018) à ce sujet, une première hypothèse incontournable consiste à stipuler que plus on perçoit l'existence et la communication d'une marque sur un réseau social donné comme légitime et justifiée de part son positionnement marketing voulu et perçu, plus on évalue favorablement le message réseau-publicitaire. 


\section{H1 : la légitimité d'existence perçue d'une réseau-publicité impacte positivement l'attitude envers la} réseau-publicité.

En alignement avec le modèle TAM, nous postulons à l'instar d'Abaidi et al. (2013) et de Herault (2012) que les cyberconsommateurs n'accepteront une réseau-publicité que si elle est jugée utile par la qualité du contenu partagé et des supports utilisés, qui elle-même est modérée par l'usage des nouvelles aides à la vente lors de la création publicitaire. Il existe subséquemment une relation positive entre la qualité perçue de la réseau-publicité et l'attitude qu'on développe à son égard.

\section{H2 : La qualité des publications impacte positivement l'attitude envers la réseau-publicité.}

H2-A: La relation entre la qualité des publications et l'attitude envers la réseau-publicité est modérée par l'usage des NAVs.

A l'instar des travaux de Maubisson et al. (2013) sur les antécédents de l'efficacité publicitaire sur facebook, les travaux d'Ezan (2014) et de Vanheems (2015) sur l'impact des messages e-publicitaires, nous stipulons que plus un cyber-annonceur exprime de l'intérêt envers son public par son discours et son comportement et s'efforce de nouer un lien avec lui, plus le feedback attitudinal et, par extension, comportemental des internautes est positif par rapport à cette communication.

H3 : L'attention portée aux internautes à travers une réseau-publicité impacte positivement l'attitude envers la réseau-publicité.

Lors d'une analyse en aval de la publicité sur réseau social, RP Bagozzi (2007) ressort avec un dernier antécédent à la réseau-publicité, à savoir la qualité de réponse aux internautes. En Effet un individu qui émet un avis, une plainte ou une question par rapport à une communication d'une marque $\mathrm{X}$ sur un réseau social $\mathrm{Y}$, et qui retrouve une réponse appropriée et rapide, développe une attitude favorable vis-à-vis de ladite réseaupublicité.

H4 : la qualité de réponse aux internautes par rapport à une réseau-publicité impacte de manière positive l'attitude envers la réseau-publicité.

En congruence avec la théorie de l'action raisonnée de Fishbein et Adjzen (1975) mais également le modèle de la hiérarchie des effets et le modèle de Herault (2012), il est supposé que plus l'individu développe une attitude positive envers une publicité en général et une réseau-publicité en particulier, plus il est enclin de la mobiliser de quelque manière que ce soit (cliquer dessus, la liker, l'enregistrer, la partager etc.).

H5 : l'attitude envers la réseau-publicité exerce une influence positive sur l'efficacité réseaupublicitaire.

Ainsi Comme sur le construit, notre propre modèle de recherche reflète par ses deux premiers éléments exogènes, la légitimité d'existence et la qualité des publications, la dimension cognitive de ce modèle. La dimension relationnelle s'incorpore dans l'attention portée aux internautes et finalement la dimension structurelle dans la qualité de réponse aux internautes.

Ainsi, nous présentons notre modèle de recherche conceptuel primaire : 


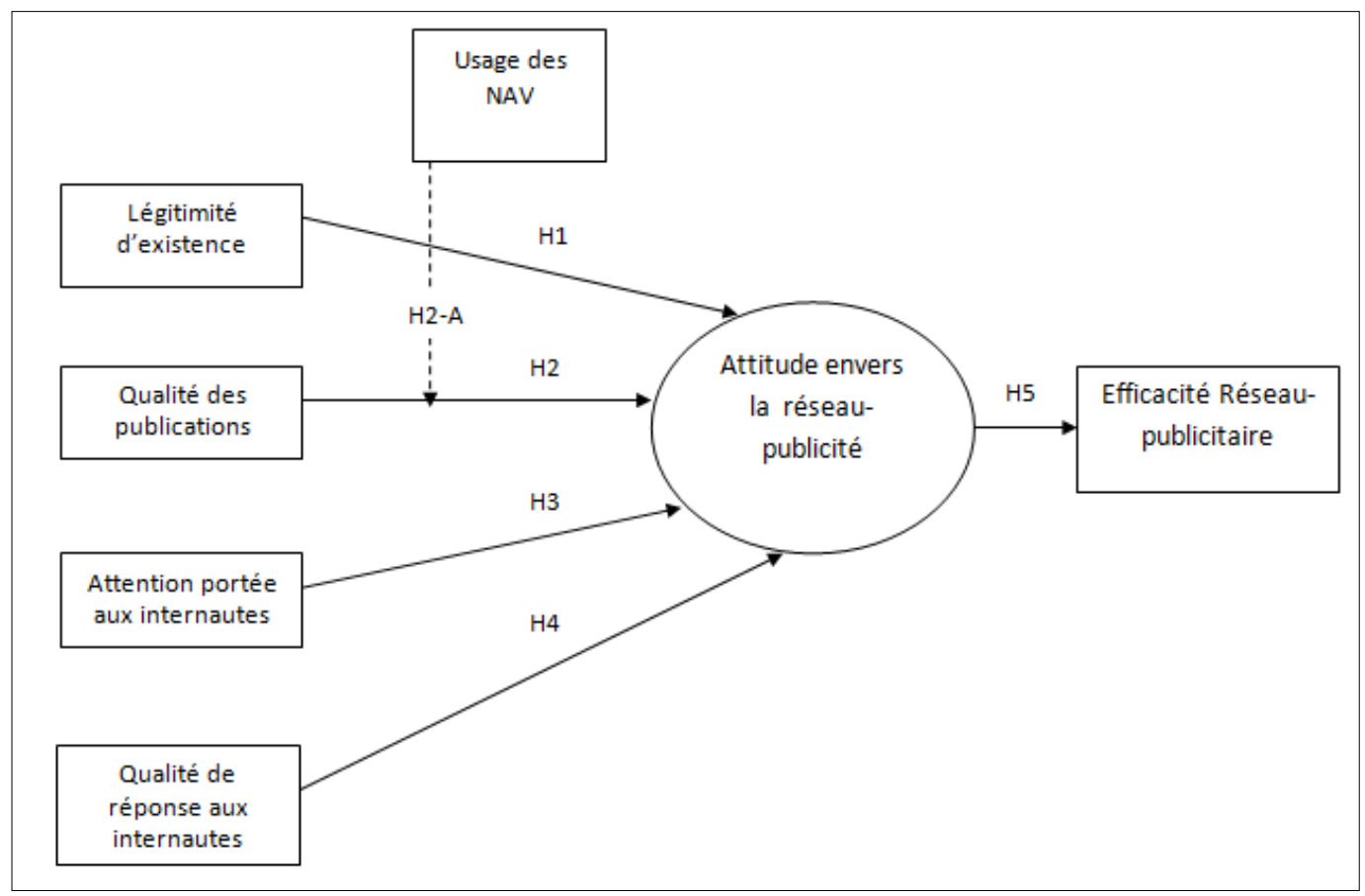

Figure 5. Représentation de notre modèle conceptuel

\section{MÉTHOdOLOGIE DE TRAVAIL :}

A ce stade, le choix des éléments adoptés pour construire notre modèle de recherche reste relativement arbitraire, dans la mesure où rien ne nous garantit jusqu'à maintenant la validité des postulats ressortis de la littérature dans le cadre du contexte choisi, à savoir les entreprises opérant par Yield Management et sur le marché nord-africain. Pour ce faire, une étude empirique a été menée, et nous en exposons la méthodologie sur ce qui suit.

Notre étude qualitative poursuit deux types d'objectifs :

- Objectif de nature contextuelle : Ramener nos hypothèses de départ au contexte des entreprises touristiques pratiquant la tarification dynamique et opérant sur le marché nord-africain.

- Objectif de nature exploratoire : collecter de nouvelles informations pour peaufiner le modèle construit sur la base de la littérature.

Une étape fondamentale de notre travail de recherche sur les antécédents de l'efficacité réseau publicitaire des entreprises pratiquant la tarification dynamique, était la contextualisation. En effet, la propriété manichéenne de notre problématique nous a incité à adopter une posture dualiste sur le terrain. Cette approche exploratoire dyadique prend tout d'abord en considération les établissements pratiquant le Yield management. Ce choix émane du fait que l'efficacité publicitaire est différemment approchée et définie par les entreprises qui ici, incarnent le rôle de l'expert. Cette démarche d'entretien avec les experts se distingue par la prise en compte d'autres types de répondants, dit experts, que ceux réellement concernés par la problématique (Malhotra, 2011). Cette technique permet un point de vue non seulement différent, mais complémentaire, et une compréhension plus profonde des dimensions étudiées (Vermersch, 2006). L'adoption de cette technique se justifie par la nature elle-même de notre problématique sur l'efficacité réseau-publicitaire. Il est vrai que nous nous intéressons à l'efficacité perçue par les cyberconsommateurs, mais nous sommes convaincus que 
l'examen de l'autre extrémité de cette relation duale entre annonceurs (les entreprises) et récepteurs (les internautes) nous a permis d'affiner d'abord le guide d'entretien utilisé à destination de la cible principale, et puis d'affiner aussi nos résultats et de ressortir les éléments les plus congruents à la construction d'un modèle de recherche adapté à notre problématique initiale.

Pour réaliser cette étude, nous avons respecté le principe de la saturation sémantique (Strauss, 1967). 12 entretiens ont été menés auprès de yield-managers, community managers, cadres marketing et cadres commerciaux de 12 entreprises marocaines et/ou nord-africaines et appartenant à trois secteurs d'activité différents à savoir, l'hôtellerie, la restauration et le transport aérien. 18 autres entretiens ont été menés avec des internautes d'âge et de CSP variés de nationalités marocaine et tunisienne disposant d'en moins un compte R.S. et étant membre confirmée d'en moins une communauté OTAs (online travel agencies) des leaders du marché mondial à savoir Booking; TripAdvisor; Trivago et Google Destination. Dans ce sens, Lemoine (2014) avance qu'un qualitatif en marketing, de 12 à 15 personnes est considéré comme bien réalisé et qu'audelà de ce seuil, « le qualitatif est considéré de très bonne qualité ».

Par ailleurs, tous les entretiens ont été menés en face à face et ont duré entre 30 à 45 minutes avec notre cible « experte », et entre 50 à 60 minutes avec les internautes. Ils se sont déroulés dans les lieux de travail respectifs de la première cible, et dans des lieux divers pour le deuxième segment de notre cible. Chaque entretien a été enregistré et retranscrit intégralement en respectant les normes de Maulini (2008).

L'analyse de contenu, qu'est un procédé dont l'objectif est d'assurer un maximum d'objectivité et de fiabilité en ce qui est de la présentation des discours des interviewés, a été adoptée pour traiter les données qualitatives collectées. La procédure d'analyse adoptée est la même pour chaque type d'entretien (entreprise et internautes) bien qu'ils soient traités séparément dans le but de comparer les résultats obtenus. Une analyse thématique a été réalisée, respectant ainsi les phases d'analyse verticale et horizontale (Giannelloni et Vernette, 2001). Pour le premier segment de notre échantillon (experts), nous avons choisi de traiter distinctivement chaque question de chaque thème principal, c.à.d. d'octroyer à chaque question une grille d'analyse indépendante où l'on retrouve les trois secteurs d'activité (hôtellerie, restauration, transport aérien) distingué l'un de l'autre. Ce choix émane du fait que nous veillons à réduire au maximum tous biais possible, dans ce cas les biais susceptibles de résulter de la nature de chaque métier et des aléas de chaque secteur d'activité.

En ce qui est du deuxième segment de notre cible (internautes), l'analyse verticale, est employée pour identifier les chemins d'accès à la formation d'une attitude favorable vis-à-vis d'une communication émise sur un réseau social par le type d'entreprise concerné, et l'analyse horizontale des corpus a permis de « mettre en exergue les constances et les régularités » (au sens de Bardin, 2003) propres à chaque segment d'une part (raisonnement intra-segment), puis en comparaison de ces segments experts et internautes (raisonnement inter-méthodes).

Voici un petit aperçu du profil de notre cible principale : 
Hathout et al.: Intention d'usage des messages réseau-publicitaires par le cybernaute nord-africain...

Tableau 1 : Récapitulatif des profils interrogés de la cible principale.

\begin{tabular}{|c|c|c|c|}
\hline Caractéristiques & Modalités & Nombre & Pourcentages \\
\hline \multirow{5}{*}{ Age } & $18-24$ & 3 & $16,7 \%$ \\
\hline & $25-34$ & 10 & $55,6 \%$ \\
\hline & $35-44$ & 1 & $5,6 \%$ \\
\hline & $45-54$ & 3 & $16,7 \%$ \\
\hline & $55-64$ & 1 & $5,6 \%$ \\
\hline \multirow{2}{*}{ Genre } & Féminin & 11 & $61 \%$ \\
\hline & Masculin & 7 & $39 \%$ \\
\hline \multirow{3}{*}{ Niveau de formation } & $\mathrm{Bac}$ à $\mathrm{Bac}+2$ & 2 & $11 \%$ \\
\hline & $\mathrm{Bac}+3$ à $\mathrm{Bac}+5$ & 12 & $67 \%$ \\
\hline & Plus que $\mathrm{Bac}+5$ & 4 & $22 \%$ \\
\hline \multirow{3}{*}{$\begin{array}{c}\text { Durée d'usage des réseaux } \\
\text { sociaux }\end{array}$} & $1-4$ ans & 5 & $28 \%$ \\
\hline & $5-8$ ans & 8 & $44 \%$ \\
\hline & plus que 8 ans & 5 & $28 \%$ \\
\hline \multirow{4}{*}{$\begin{array}{l}\text { Fréquence d'usage des } \\
\text { réseaux sociaux }\end{array}$} & Quotidiennement & 11 & $61 \%$ \\
\hline & Régulièrement & 7 & $39 \%$ \\
\hline & Occasionnellement & 0 & $0 \%$ \\
\hline & Rarement & 0 & $0 \%$ \\
\hline \multirow{4}{*}{ lieux d'accès } & Domicile & 7 & $39 \%$ \\
\hline & Travail & 4 & $22 \%$ \\
\hline & Téléphone & 1 & $6 \%$ \\
\hline & $\begin{array}{l}\text { Lieu public (Cafés, } \\
\text { Facultés, hôtels...) }\end{array}$ & 7 & $39 \%$ \\
\hline
\end{tabular}

\section{RÉSULTATS DE L'ÉTUDE QUALITATIVE :}

A travers notre analyse thématique horizontale, nous avons pu dresser, pour chacune des chaires de notre échantillon une grille d'analyse synthétique ressortant le nombre et la fréquence de citation de chaque dimension : 
Tableau 2 : Grille d'analyse synthétique auprès des internautes.

\begin{tabular}{|c|c|c|}
\hline Dimensions & $\begin{array}{c}\text { Nombre de } \\
\text { citation }\end{array}$ & $\begin{array}{c}\text { \% des répondants ayant } \\
\text { évoqués la dimension }\end{array}$ \\
\hline Légitimité d'existence & 5 & $5 / 18=28 \%$ \\
\hline Qualité des publications & 88 & $18 / 18=100 \%$ \\
\hline $\begin{array}{c}\text { Usage des NAVs (qualité } \\
\text { des publications) }\end{array}$ & 87 & $18 / 18=100 \%$ \\
\hline $\begin{array}{c}\text { Attention portée aux } \\
\text { internautes }\end{array}$ & 78 & $18 / 18=100 \%$ \\
\hline $\begin{array}{c}\text { Qualité de réponse aux } \\
\text { internautes }\end{array}$ & 73 & $18 / 18=100 \%$ \\
\hline $\begin{array}{c}\text { L'attitude envers la } \\
\text { réseau-publicité }\end{array}$ & 71 & $18 / 18=100 \%$ \\
\hline $\begin{array}{c}\text { L'efficacité réseau- } \\
\text { publicitaire }\end{array}$ & 63 & $18 / 18=100 \%$ \\
\hline $\begin{array}{c}\text { Délai de réponse (Qualité } \\
\text { de réponse aux } \\
\text { internautes) }\end{array}$ & 29 & \\
\hline
\end{tabular}

Tableau 3 : Grille d'analyse synthétique auprès des experts

\begin{tabular}{|c|c|c|}
\hline Dimensions & $\begin{array}{c}\text { Nombre } \\
\text { de citation }\end{array}$ & $\begin{array}{c}\text { \% des répondants ayant } \\
\text { évoqués la dimension }\end{array}$ \\
\hline Légitimité d'existence & 9 & $5 / 8=62.5 \%$ \\
\hline Qualité des publications & 43 & $8 / 8=100 \%$ \\
\hline Usage des NAVs & 18 & $7 / 8=87.5 \%$ \\
\hline $\begin{array}{c}\text { Attention portée aux } \\
\text { internautes }\end{array}$ & 24 & $7 / 8=87.5 \%$ \\
\hline $\begin{array}{c}\text { Qualité de réponse aux } \\
\text { internautes }\end{array}$ & 49 & $8 / 8=100 \%$ \\
\hline $\begin{array}{c}\text { L'attitude envers la réseau- } \\
\text { publicité }\end{array}$ & 22 & $8 / 8=100 \%$ \\
\hline $\begin{array}{c}\text { L'efficacité réseau- } \\
\text { publicitaire }\end{array}$ & 33 & $8 / 8=100 \%$ \\
\hline
\end{tabular}

Ceci nous a permis d'effectuer une hiérarchisation des variables en se basant sur l'intérêt perçu de toute une chacune d'entre elles. A coté de ceci, nous avons pu allouer à chaque variable une définition contextuelle traduisant la perception des répondants : 
Hathout et al.: Intention d'usage des messages réseau-publicitaires par le cybernaute nord-africain...

Tableau 4 : Hiérarchisation verticale de toutes les variables selon les résultats de la cible cybernautes usagers de réseaux sociaux.

\begin{tabular}{|c|c|c|}
\hline Rang & Dimensions & Définitions \\
\hline 1 & Qualité des publications & $\begin{array}{l}\text { C'est la capacité du message réseau- } \\
\text { publicitaire à miroiter l'expérience qu'est la } \\
\text { prestation. }\end{array}$ \\
\hline 2 & $\begin{array}{l}\text { Usage des NAVs (se rapporte à la qualité } \\
\text { des publications) }\end{array}$ & $\begin{array}{l}\text { C'est la capacité du message réseau- } \\
\text { publicitaire à stimuler le côté sensoriel et affectif } \\
\text { de l'internaute }\end{array}$ \\
\hline 3 & Attention portée aux internautes & $\begin{array}{l}\text { C'est l'intérêt perçu que confère les réseau- } \\
\text { annonceurs à leur audience digitale. }\end{array}$ \\
\hline 4 & Attitude envers la réseau-publicité & $\begin{array}{l}\text { Se rapporte à la réaction affective de } \\
\text { l'internaute à l'égard de la publicité (réaction } \\
\text { positive ou négative) }\end{array}$ \\
\hline 5 & Qualité de réponse aux internautes & $\begin{array}{l}\text { Renvoie au fait que l'internaute se sent écouté } \\
\text { et reçoit un feedback pertinent et engageant } \\
\text { (captivant) }\end{array}$ \\
\hline 6 & $\begin{array}{l}\text { Délai de réponse (se rapporte à la qualité } \\
\text { de réponse aux internautes) }\end{array}$ & $\begin{array}{l}\text { C'est la capacité du réseau-annonceurs à } \\
\text { répondre aux interventions des internautes avant } \\
\text { le désintérêt de ces derniers }\end{array}$ \\
\hline 7 & Efficacité réseau-publicitaire & $\begin{array}{l}\text { Reflète la disposition des internautes à utiliser } \\
\text { la réseau-publicité de quelque manière que ce soit } \\
\text { (liker, partager, envoyer, enregistrer...) }\end{array}$ \\
\hline
\end{tabular}

En analysant ces résultats, des constats phares nous permettent de remanier nos hypothèses et notre modèle. Ainsi, et en congruence avec ce qui a été retrouvé lors de notre première analyse horizontale (experts) la dimension «légitimité d'existence » a été rejeté par notre cible principale qui affirme qu'aujourd'hui aucune marque n'a besoin de justifier sa présence ou sa communication sur les R.S. quelque soit son positionnement Marketing, la nature de son activité ou de ses produits. A l'exception de la proposition se rapportant au réseau-positionnement, les autres dimensions relevées de la revue de littérature ont été citées par l'ensemble des interviewés, et sont fortement confirmées par ces derniers. D'entre les dimensions confirmées, un nouvel élément fait surface, celui du «délai de réponse ». En effet il a été confirmé comme un élément modérateur entre la qualité de réponse aux internautes et l'attitude envers la réseau-publicité. A ce niveau un retour vers la littérature était nécessaire pour confirmer l'importance de ce nouvel élément. En effet, il s'est avéré qu'elle regorge de références académiques et statistiques attestant de la portée de cette variable et qui nous a apparemment échappé lors de la construction de notre modèle primaire. Et finalement, en ce qui est de notre variable endogène que nous avons baptisé «efficacité réseau-publicitaire », elle passe dans notre cadre et pour notre cible principale par «l'intention d'usage » des messages publiés par les annonceurs sur leurs 
pages R.S. (visionner, partager, liker...). Un résultat qui va de paire avec le modèle TAM qui cite l'intention comportementale d'usage.

Ainsi nos hypothèses et modèle de recherche deviennent comme suite :

Tableau 5 : Tableau récapitulatif des hypothèses

\begin{tabular}{|c|c|}
\hline \multicolumn{2}{|r|}{ Hypothèses de la recherche } \\
\hline H1 & $\begin{array}{c}\text { La qualité des publications impacte de manière positive l'attitude envers la réseau- } \\
\text { publicité. }\end{array}$ \\
\hline H1-A & $\begin{array}{l}\text { La relation entre la qualité des publications et l'attitude envers la réseau-publicité } \\
\text { est modérée par l'usage des NAV. }\end{array}$ \\
\hline H2 & $\begin{array}{l}\text { L'attention portée aux internautes impacte de manière positive l'attitude envers la } \\
\text { réseau-publicité. }\end{array}$ \\
\hline $\mathbf{H 3}$ & $\begin{array}{l}\text { La qualité de réponse aux internautes impacte de manière positive l'attitude envers } \\
\text { la réseau-publicité. }\end{array}$ \\
\hline H3-A & $\begin{array}{l}\text { La relation entre la qualité de réponse aux internautes et l'attitude envers la réseau- } \\
\text { publicité est modérée par le délai de réponse. }\end{array}$ \\
\hline H4 & l'attitude envers la réseau-publicité influence l'intention d'usage. \\
\hline
\end{tabular}

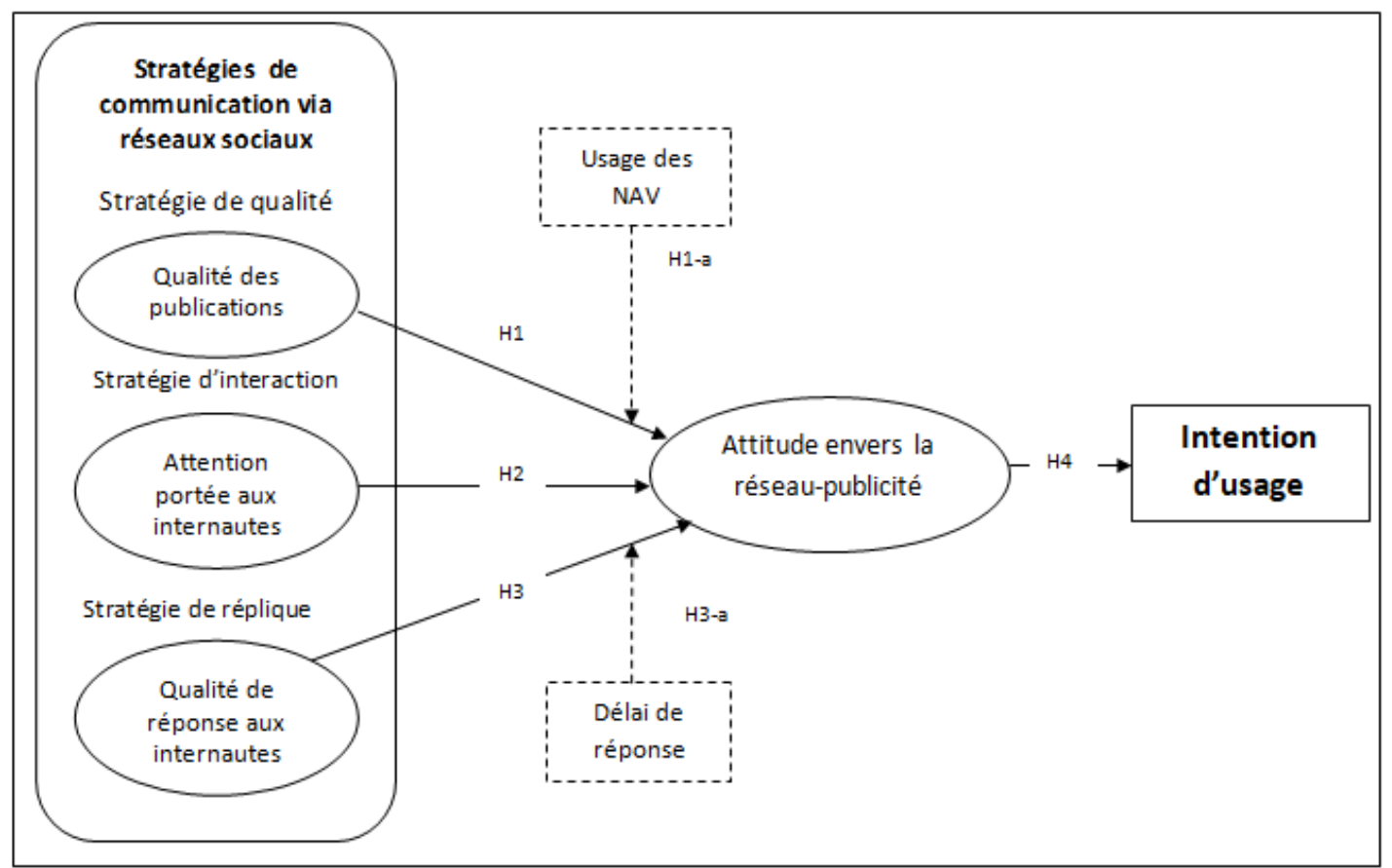

Figure 6. Représentation de notre modèle conceptuel après contextualisation qualitative.

Nous remarquons le rejet de la lére hypothèse et donc la suppression d'une variable explicative qu'est la légitimité d'existence, l'ajout d'un nouvel élément modérateur qu'est le délai de réponse entre la qualité de réponse aux internautes et l'attitude et puis le changement de notre variable endogène qui devient dés lors intention d'usage des messages réseau-publicitaires. 
On obtient alors un modèle testé qui suppose que l'efficacité des messages réseau-publicitaires traduite dans notre contexte par l'intention d'usage passe par l'attitude que développe la communauté envers ledit message, qui elle-même est conditionnée par le comportement de la marque annonciatrice :

- En amont de la création publicitaire en soignant la qualité des publications émises sur les réseaux sociaux et qui passe par un contenu congruent aux attentes des cybernautes et des supports innovants par l'usage des NAVS. D'un autre côté en exprimant un intérêt particulier envers cette communauté de part les thèmes traités, l'écoute dont fait preuve la marque et les avantages commerciaux offerts en ligne.

- En aval de la publication par la qualité des réponses aux interventions des internautes qui devraient être pertinentes et rapides avant le désintérêt des intervenants.

\section{CONCluSion :}

Il ne nous reste plus à ce niveau que de finir sur les limites, apports et perspectives de notre recherche.

En ce qui est des apports de notre recherche, ils se scindent en 3 catégories :

- Le premier apport, qui est d'ordre théorique, passe par la proposition d'un modèle qui prédit que la mobilisation des stratégies de communication dans un contexte « digi-social » transforme la nature même des éléments sur lesquels le cybernaute se base pour développer l'intention d'usage d'un message réseau-publicitaire.

- Sur le plan méthodologique, notre travail se distingue par la méthode de traitement unitaire des questions ayant fait l'objet, tout une chacune, d'une grille d'analyse indépendante ressortant les trois secteurs d'activités étudiés.

- Et finalement d'un point de vue managérial, les résultats de notre travail de recherche viennent apporter quelques éléments de réponse susceptibles d'assister les managers dans la création et la diffusion d'un contenu efficace sur leurs pages R.S.. On pense avoir mis à la disposition de nos managers des outils susceptibles de les assister dans le Pilotage de l'efficacité de la réseaucommunication et aussi la gestion des dimensions et des antécédents de l'intention d'usage des messages réseau-publicitaires chez l'internaute social nord-africain.

Il est connu que tout travail de recherche renferme des limites, et notre cas ne fait pas l'exception. En effet les grandes limites de notre travail de recherche se scient en 3 catégories :

- D'abord des limites relatives au contexte de la recherche qui demeure restreint par rapport à l'amplitude de la problématique. Les résultats obtenus ne sont valides que pour une chaire bien déterminée d'entreprises, à savoir celle opérant par la tarification dynamique et sur les secteurs d'hébergement, de restauration et de transport aérien.

- Limites relatives au développement technologique et l'essor ascendant des nouvelles technologies de communication, des objets connectées, des canaux de communication sociale en général et des medias sociaux en particulier, des pratiques managériales, du comportement des géants du net (Exemple de la GAFAM: Google, Amazon, Facebook et Apple), du comportement des internautes, et de tout ce qui pourrait s'en suivre. Ces situations sont susceptibles de rendre, progressivement ou promptement, obsolètes les résultats d'une telle recherche. 
- Limites relatives au cadre contextuel : Un choix a été fait pour considérer le Yield management exclusivement comme étant le contexte empirique de notre étude et non pas une variable à intégrer dans le modèle de recherche. Ainsi, l'effet des attributs du Yield management n'apparait pas directement mais reste imbriqué à la structure globale du modèle.

En ce qui est des perspectives de notre recherche, on propose :

- Une extension du terrain d'étude et un enrichissement possible du modèle. En effet, il serait intéressant de vérifier la validité de notre modèle dans des contextes différents ou plus étendus :

$\checkmark$ Étendu géographique : aller au-delà des frontières nord-africaines pour répliquer ce travail dans des zones culturellement similaires à la nôtre ou complètement différentes.

$\checkmark$ Contextualisation sectorielle : Etendre les résultats de ce travail à d'autres secteurs opérant par la tarification dynamique (Ex : prestations de téléphonie), ou carrément hors Yield management.

$\checkmark$ Cadre conceptuel : Dans le cadre de cette recherche, nous nous sommes abstenus à l'étude de l'efficacité publicitaire sur les réseaux sociaux. A cet effet, il serait intéressant de pousser les bordures pour inclure l'une ou plusieurs des 5 autres catégories de médias sociaux (selon la pyramide de Mooc-Coursera) comme les blogs, les newsletters, etc. ...

$\checkmark$ On pourrait aussi chercher à enrichir notre modèle conceptuel en intégrant d'autres variables à notre modèle. Des variables susceptibles d'enrichir le sens holistique de nos résultats.

- Quoique notre cible principale d'internautes sociaux associe exclusivement l'efficacité réseaupublicitaire à l'intention d'usage, les professionnels (cible experte) citent bel et bien la résultante comportementale comme élément exprimant l'efficacité publicitaire des messages émis sur les réseaux sociaux pour les entreprises opérant par tarification dynamique sur le marché nordafricain. A cet effet, il serait judicieux et intéressant de pouvoir examiner l'effet qu'aura l'introduction de cette dimension comportementale sur la structure de notre modèle.

- Enfin, il est vrai que nous avons effectué le choix de considérer le Yield management exclusivement comme contexte empirique de l'étude et non pas une variable à intégrer dans le modèle de recherche. Ceci a permis d'examiner l'effet des pratiques propre à ce système sur les construits de notre modèle mais de façon sous-jacente. A ce stade nous considérons que notre modèle peut être enrichi et étoffé grâce à l'introduction des composantes et/ou attributs du Yield management qui peuvent faire, par exemple, l'objet de variables modératrices.

\section{BIBLIOGRAPHIE :}

[1] Abaidi, I., \& Vernette, E. (2018). Does digitalization create or reduce perceived global value? Journal of Consumer Marketing.

[2] Abdelkefi, M. I., \& Ben Brahim, S. (2015). Attitude à l'égard de la publicité mobile et intention de l'utiliser par les Tunisiens,'. International Journal of Economics \& Strategic Management of Business Process, 5, 1-6.

[3] Ajzen, I., \& Fishbein, M. (1975). A Bayesian analysis of attribution processes. Psychological bulletin, 82(2), 261.

[4] Anderson, E. K., Aberle, E., Chen, C., Egenolf, J., Harmoney, K., Kakani, V. G., ... \& Lee, D. (2016). Impacts of management practices on bioenergy feedstock yield and economic feasibility on Conservation Reserve Program grasslands. Gcb Bioenergy, 8(6), 1178-1190.

[5] Bagozzi, R. P. (2007). The legacy of the technology acceptance model and a proposal for a paradigm shift. Journal of the association for information systems, 8(4), 3 . 
[6] Bagozzi, R. P. (2007). The legacy of the technology acceptance model and a proposal for a paradigm shift. Journal of the association for information systems, 8(4), 3 .

[7] Barber, P., \& Wallace, L. (2010). Building a buzz: libraries \& word-of-mouth marketing. American Library Association.

[8] Bardin, L. (2003), L'analyse du contenu. 7ème. Paris: PUF

[9] Bauer, H. H., Reichardt, T., Barnes, S. J., \& Neumann, M. M. (2005). Driving consumer acceptance of mobile marketing: A theoretical framework and empirical study. Journal of electronic commerce research, 6(3), 181.

[10] Beck, M., \& Crié, D. (2018). I virtually try it... I want it! Virtual Fitting Room: A tool to increase on-line and off-line exploratory behavior, patronage and purchase intentions. Journal of Retailing and Consumer Services, 40, 279-286.

[11] Benmoussa, F. Z., Errajaa, K., Maubisson, L., \& Maynadier, B. (2015). Les mécanismes de la co-production d'une expérience: une approche par la dimension sensible. Management Avenir, (3), 71-91.

[12] Blal, I., \& Sturman, M. C. (2014). The differential effects of the quality and quantity of online reviews on hotel room sales. Cornell Hospitality Quarterly, 55(4), 365-375.

[13] Boucher, B., Girginov, V., Taks, M., Martyn, S., Holman, M., \& Dixon, J. (2009). Canadian national sport organizations' use of the web for relationship marketing in promoting sport participation. International Journal of Sport Communication, 2(2), 164-184.

[14] Bousquet, F., Marty, E., \& Smyrnaios, N. (2015). Les nouveaux acteurs en ligne de l'information locale vers une relation aux publics renouvelée?. Sur le journalisme, About journalism, Sobre jornalismo, 4(2), 48-61.

[15] Capiez, A. (2003). Yield management: optimisation du revenu dans les services. Hermès Science.

[16] Cooper, W. L., Homem-de-Mello, T., \& Kleywegt, A. J. (2006). Models of the spiral-down effect in revenue management. Operations research, 54(5), 968-987.

[17] Courbet, D., \& Fourquet-Courbet, M. P. (2005). Modèles et mesures de l'influence. Revue internationale de psychosociologie, 11(25), 171-195.

[18] Ezan, P., Gollety, M., Guichard, N., \& Hémar-Nicolas, V. (2014). Renforcer l'efficacité des actions de lutte contre l'obésité. Vers l'identification de leviers de persuasion spécifiques aux enfants. Décisions Marketing, 13-26.

[19] Fogel, S. (2014). Issues in measurement of word of mouth in social media marketing. International Journal of Integrated Marketing Communications, 2(2).

[20] Giannelloni, J. L., \& Vernette, E. (2001). Etudes de marché, 2 e éd. Vuibert, Paris, 226-249.

[21] Hérault, S. (2010). Mesure de l'efficacité de la publicité mobile: un essai de modélisation intégrant le caractère intrusif et l'utilité perçue de la publicité mobile. Université de Paris, 1.

[22] LEMOINE, J. F. CHERIF, E., \& (2014). L'influence du type de voix du conseiller virtuel sur la présence sociale, la confiance envers le conseiller virtuel, la confiance envers le site et les inten-tions comportementales. In 30 ème Colloque International de l'Association Française du Marketing.

[23] Malhotra, D., \& Lumineau, F. (2011). Trust and collaboration in the aftermath of conflict: The effects of contract structure. Academy of management Journal, 54(5), 981-998.

[24] Maulini, O., \& Perrenoud, P. (2008). Sciences sociales et savoirs d'expérience: conflit de questions ou conflits de réponses. Conflits de savoirs en formation des enseignants. Entre savoirs issus de la recherche et savoirs issus de l'expérience, 141-153.

[25] Noone, B. M., McGuire, K. A., \& Rohlfs, K. V. (2011). Social media meets hotel revenue management: Opportunities, issues and unanswered questions. Journal of Revenue and Pricing Management, 10(4), 293-305.

[26] Ranchoux, C., David, M., \& Maubisson, L. (2016). La valeur perçue des outils de communication interne: implications théoriques et managériales (No. hal-02499748).

[27] Sago, B. (2011). The Usage Level and Effectiveness of Quick Response (QR) Codes for Integrated Marketing Communication Purposes among College Students. International Journal of Integrated Marketing Communications, 3(2).

[28] Sallard I. (2007), Comment faire du marketing direct écologique, Marketing Direct, $n^{\circ} 110$. 
[29] Sigala, M., Christou, E., \& Gretzel, U. (Eds.). (2012). Social media in travel, tourism and hospitality: Theory, practice and cases. Ashgate Publishing, Ltd.

[30] Strauss, A., \& Corbin, J. (1967). Discovery of grounded theory.

[31] Thiers, B. (2017). Social network analysis. Sage.

[32] Tourigny, m., baillargeon, d., carignan, m., rocheleau, s., \& vincent, n. (2020). Des journalistes, à l'ére de la publicité native. département de communication faculté des lettres et sciences humaines université de sherbrooke.

[33] Valin, P., Blasch, E. P., Lambert, D. A., Kokar, M. M., Llinas, J., Das, S., ... \& Shahbazian, E. (2012). High level information fusion (hlif): Survey of models, issues, and grand challenges. IEEE Aerospace and Electronic Systems Magazine, 27(9), 420.

[34] Vanheems, R., \& Paché, G. (2018). La distribution face au consommateur connecté: un monde au bout des doigts... et après?. Décisions marketing, (3), 5-21.

[35] Venkatesh, V., Morris, M. G., Davis, G. B., \& Davis, F. D. (2003). User acceptance of information technology: Toward a unified view. MIS quarterly, 425-478.

[36] Vermersch, P. (2006). L'ENTRETIEN.

[37] Wirtz, J., \& Lovelock, C. H. (2005). Services marketing in Asia: A case book. Prentice Hall.

[38] Zlitni, S., \& Liénard, F. (2012). La communication électronique comme élément de la trace numérique. Netcom. Réseaux, communication et territoires, (26-1/2), 9-14

[39] Zrelli, I. (2010). Les déterminants de l'orientation Yield Management. Revue française de gestion, (8), 63-82. 